Cahiers de recherches médiévales

Nouvel Énée, ou faux Énée?

Jules César et Eneas dans le Roman de Jules César de Jean de Thuin

\title{
Francine Mora
}

\section{(2) OpenEdition}

Journals

Édition électronique

URL : https://journals.openedition.org/crm/2574

DOI : $10.4000 / \mathrm{crm} .2574$

ISSN : $1955-2424$

Éditeur

Honoré Champion

Édition imprimée

Date de publication : 30 juin 2007

Pagination : 175-189

ISSN : 1272-9752

Référence électronique

Francine Mora, « Nouvel Énée, ou faux Énée? », Cahiers de recherches médiévales [En ligne], 14 spécial | 2007, mis en ligne le 30 juin 2010, consulté le 15 décembre 2022. URL : http://

journals.openedition.org/crm/2574; DOI : https://doi.org/10.4000/crm.2574 


\title{
ris
}

\section{Nouvel Énée, ou faux Énée ? Jules César et Eneas dans le Roman de Jules César de Jean de Thuin}

\begin{abstract}
Depuis son édition récente par Olivier Collet, qui lui a également consacré une étude détaillée, le Roman de Jules César de Jean de Thuin retient de plus en plus souvent l'attention de la critique'. Il offre en effet l'exemple intéressant d'une «mise en roman» doublement atypique : d'abord parce qu'elle est écrite, comme le Roman d'Alexandre d'Alexandre de Paris, en laisses d'alexandrins, ce qui l'apparente à une chanson de geste, ensuite parce qu'elle est très tardive, ayant sans doute été composée dans les années 1260-1270, ce qui la rend contemporaine des poèmes de Rutebeuf et du Roman de la Rose de Jean de Meun². Pourtant les principes qui sont énoncés dans son prologue, comme l'a bien montré Olivier Collet, reprennent assez fidèlement ceux des premières «mises en roman» du milieu du XII ${ }^{\mathrm{e}}$ siècle : au projet de transférer une riche «matere » «du latin toute en romanz » à partir de la Pharsale de Lucain s'associe dès les premiers vers un désir de «trover» qui affirme d'emblée que l'adaptateur, qui prétend au statut d' «auctors», ne s'interdira pas toute invention poétique ${ }^{3}$. Par rapport aux Faits des Romains dont il s'inspire aussi, le roman de Jean de Thuin apparaît ainsi comme la réécriture active et orientée d'une œuvre antique plus que comme une compilation historique, à l'instar des romans de Thèbes, d'Eneas et de Troie, ce qui lui permet du reste d'offrir de son héros une image beaucoup plus méliorative que celle que proposait Lucain. Par ailleurs Jean de Thuin, comme a pu le montrer aussi Olivier Collet, connaissait sans doute Eneas et Troie, auxquels renvoient quelques allusions discrètes mais suggestives placées intentionnellement à des endroits stratégiques de son récit. Ainsi le séjour de César à Alexandrie, qui amène la rencontre avec Cléopâtre, est précédé comme chez Lucain par une visite aux ruines de Troie ; mais le récit de cette visite est accompagné d'un rappel détaillé du jugement de Pâris, ce qui n'est pas le cas dans la Pharsale, qui se contente d'une très brève allusion à «l'antre où siégea l'arbitre », et ce rappel, qui évoque bien sûr le Roman de Troie, est placé de manière surprenante sous l'autorité de Virgile, ce qui semble vouloir renvoyer implicitement à l'entrée en matière narrative de l'Eneas. Cela d'autant mieux que le nom d'Eneas, présenté comme l' «ancestres» de César, est mentionné deux fois dans la laisse qui précède celle du jugement de Pâris, que ce nom est
\end{abstract}

\footnotetext{
${ }^{1}$ Jean de Thuin, Le Roman de Jules César, éd. O. Collet, Genève, Droz (TLF), 1993 et O. Collet, Étude philologique et littéraire sur le Roman de Jules César, Genève, Droz, 1993.

${ }^{2}$ Jean de Thuin, Le Roman de Jules César, éd. cit., p. XI.

${ }^{3}$ Jean de Thuin, Le Roman de Jules César, éd. cit., v. 2, 32-34, 63 et O. Collet, Étude..., op. cit., p. 95-100 (« faire romanz nouvelement (v. 13) : le projet littéraire de l'auteur »).

${ }^{4}$ Lucain, La guerre civile (La Pharsale), éd. et trad. A. Bourgery et M. Ponchont, Paris, Les Belles Lettres, 1974, livre IX, v. 971 (quo iudex sederit antro). Le mot «jugierres », qui qualifie Pâris au v. 5941 du Roman de Jules César, semble un écho direct du iudex de Lucain. Mais le demi-vers de l'épopée antique s'est développé en une laisse entière dans le roman médiéval.
}

Cahiers de Recherches Médiévales, 14spé, 2007 
apparu dès le vers 842 à propos de l'exil temporaire de Pompée à Capoue, et que celui de Didon est cité au moment de l'arrivée de Curion, l'un des partisans de César, à Carthage, au début de la première campagne africaine, comme si Jean de Thuin avait voulu, «sur un itinéraire pointant l'Égypte de Cléopâtre, [faire se] profile[r] l'aventure impériale d'Énée-César dans sa double exigence, amoureuse [...] et conquérante ${ }^{5} »$.

Faut-il alors voir César comme un «nouvel Énée ${ }^{6} » ?$ La suggestion est intéressante, mais mérite d'être discutée. Silvère Menegaldo, qui a analysé en détail, lors d'un précédent colloque, le héros de Jean de Thuin, a fini par conclure sur sa résistance à se muer en personnage romanesque, malgré un caractère «problématique» ou «ambivalent» qui l'apparente à Eneas et à Alexandre et qui semble donc, à première vue, le rendre particulièrement apte à ce type de transformation ${ }^{7}$. Nous voudrions à notre tour revenir sur cette question en abordant l'épisode des amours de César et de Cléopâtre, que Silvère Menegaldo a laissé de côté, mais qui selon Olivier Collet emprunte beaucoup à celui des amours d'Eneas et de Lavine, ne serait-ce qu'en raison de la présence d'un développement théorique qui s'insère au cœur de cet épisode et qui fait «coexiste[r] la fable courtoise proprement dite avec une pensée didactique inspirée par l'observation des comportements que suscite l'amour ${ }^{8} »$. Cette coexistence, de l'avis d'Olivier Collet, a été directement inspirée par l'Eneas: «comme Lavinie, écrit-il, Cléopâtre intervient au cœur d'un débat crucial pour le héros ; son apparition, concrétisant au regard de l'imaginaire romanesque l'un des aspects essentiels de l'aventure, matérialise l'union possible de militia et d' amor $^{9}{ }^{~}$. Souveraine orientale susceptible d'évoquer Didon, Cléopâtre serait donc en même temps une nouvelle Lavine, et son rôle serait de donner au conquérant cette dimension amoureuse et courtoise sans laquelle il n'est point de héros romanesque. Ce faisant Jean de Thuin s'inscrirait nettement en faux contre Lucain, qui avait stigmatisé «la honte de l'Égypte, celle dont l'impureté a fait le malheur de Rome ${ }^{10}$ », et contre l'auteur des Faits des Romains, qui parle à propos de Cléopâtre de «luxure» et de "puterie ${ }^{11}$. Il est indéniable que l'image de Cléopâtre, comme celle de César, a fait l'objet dans le roman de Jean de Thuin d'une réécriture méliorative. Mais cette réécriture ne présente-t-elle plus aucune ambivalence, et permet-elle vraiment d'assimiler la reine

\footnotetext{
${ }^{5}$ Jean de Thuin, Le Roman de Jules César, éd. cit., v. 2564, 5928, 5932 et O. Collet, Étude..., op. cit., p. 120-125 (cit. p. 121, n. 4).

${ }^{6}$ O. Collet, Étude..., op. cit., p. 121.

${ }^{7}$ S. Menegaldo, «César 'd'ire enflamez et espris' (v. 1696) dans Le Roman de Jules César de Jean de Thuin », Cahiers de Recherches Médiévales (XII $-X V^{e}$ s.), t. 13 spé., 2006, p. 59-76. Voir aussi, dans le même recueil, l'article de M. Szkilnik, «César est-il un personnage de roman? Du Perceforest au Jouvencel », p. 77-89.

${ }^{8}$ O. Collet, Étude..., op. cit., p. 130.

${ }^{9}$ Ibid.

${ }^{10}$ Lucain, La guerre civile (La Pharsale), éd. cit., livre X, v. 59-60 (dedecus Aegypti, [...]/ Romano non casta malo) (cités par O. Collet, Étude..., op. cit., p. 125).

${ }^{11}$ Les Faits des Romains, éd. L. F. Flutre et K. Sneyders de Vogel, Paris-Groningue, 1938 et Genève, Slatkine Reprints, 1977, p. 627 (cité par O. Collet, Étude..., op. cit., p. 128).
} 
d'Égypte à Lavine ? Voilà les deux questions que l'on peut se poser, et auxquelles nous allons essayer de répondre.

Comme l'a montré Joachim Leeker dans sa vaste synthèse sur $L a$ représentation de César dans les littératures romanes du Moyen Age, l'image d'un César amoureux n'est pas rare dans les œuvres poétiques des $\mathrm{XIV}^{\mathrm{e}}$ et $\mathrm{XV}^{\mathrm{e}}$ siècles $^{12}$. Il cite notamment un intéressant passage du Livre du Cour d'amour épris de René d'Anjou qui fait figurer son blason en tête de ceux des amants célèbres dans le cimetière du dieu d'Amour, avant même ceux de Pâris et d'Énée, et qui le présente en ces termes :

Je Julles, dit Cezar, d'excercite ducteur,

Et de la republique premier apprehendeur,

Puissant et redoubté et preux et conquerant,

Davant qui le mondë aloit de peur tremblant,

Vins cy jadis faisant au dieu d'Amours homage,

De treshumble vouloir et de piteux courage,

Moy rendant son subget de par Cleopatra,

La tresnoble royne d'Egipte, qui frappa

Le coup de l'ardant dart qui me navra le cueur ;

Dont lors habandonné tout pris et los d'onneur,

Conquestes et vaillances et les nobles faiz d'armes :

Pour mener vie oyzeuse ay ycy mis mes armes ${ }^{13}$.

René d'Anjou se souvient-il ici du roman de Jean de Thuin ou de son adaptation en prose, Li Hystore de Julius Cesar, œuvre du même auteur et très proche du texte en vers? Ce n'est pas impossible, car l'auto-portrait que fait ici de lui le chef romain présente bien des points communs avec l'image qu'en offre, dans l'adaptation du dixième et dernier livre de Lucain, la «mise en roman» du XIII ${ }^{\mathrm{e}}$ siècle : celle d'un conquérant redoutable, mais vaincu par l'amour, qui a démontré ainsi sa toutepuissance. Comme l'écrit Jean de Thuin,

Or a li diex d'amors esploitié come ber et si s'en puet molt bien chier tenir et venter, qu'il a navré ou cuer d'un dart qui fet amer le plus vaillant baron qu'en el mont puist trover ${ }^{14}$.

Bien qu'elle soit topique, la reprise des mêmes termes (cuer, dart, navrer) peut signaler une discrète filiation. Et derrière ce guerrier frappé par le dard de l'amour, on peut effectivement voir se profiler le souvenir d'Eneas, frappé lui aussi par le

12 J. Leeker, Die Darstellung Cäsars in den romanischen Literaturen des Mittelalters, Francfort, Klostermann, 1986, p. 355-360.

${ }^{13}$ René d'Anjou, Le Livre du Couur d'amour épris, éd. et trad. F. Bouchet, Paris, Le Livre de Poche, coll. «Lettres Gothiques », 2003, § LXXXIII, v. 1207-1218. Passage cité par J. Leeker, op. cit., p. 359, n. 53.

${ }^{14}$ Jean de Thuin, Le Roman de Jules César, éd. cit., v. 6303-6306. 
«dart d'or » de son frère, Cupido, le «deus d'amor », au moment où il lisait la lettre que Lavine lui avait envoyée enroulée autour d'une flèche :
Amors, ne ai vers toi rados,
tu ne me lais avoir repos [...].
Tu m'as de ton dart d'or navré,
mal m'a li briés anpoisoné
qu'entor la saiete trovai ${ }^{15}$.

Comme l'a fait remarquer Olivier Collet, le caractère stéréotypé des motifs ne permet guère toutefois d'établir l'existence d'une imitation directe ${ }^{16}$. Ce qui est certain, cependant, c'est que tout est mis en œuvre par Jean de Thuin pour faire de son héros un digne émule du «fin amant » exemplaire mis en scène soit par Eneas et Troie, soit par des textes postérieurs comme les romans de Chrétien de Troyes ${ }^{17}$. Frappé par l'amour, César s'abîme dans la pensée de sa dame au point de bannir toute autre pensée, notamment guerrière, et connaît les tourments de l'insomnie :

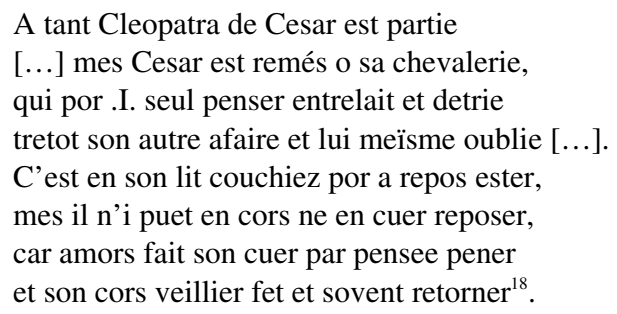

Soucieux de faire connaître sa passion avec délicatesse, il choisit ensuite de recourir à la médiation d'un «chevaliers cortois » (v. 6787) qui se fait son porte-parole pour déclarer son amour à Cléopâtre, car cet amour est si intense que mis en présence de sa belle, César demeure muet :
Lors en a tel vergoigne que il ne set coment ne de qoi aresnier la puist premierement, ainz li faut tot et serre li cuers outreement por l'amor qui son cuer esbahist et sorprent ${ }^{19}$.

Le mutisme et la timidité du héros, qui avait su pourtant, lors d'une première entrevue, dire à Cléopâtre toute l'admiration qu'il lui portait, rendent ici témoignage

\footnotetext{
${ }^{15}$ Eneas, éd. J. J. Salverda de Grave, Paris, Champion (CFMA), 1925 et 1929, t. II, v. 89498955.

${ }^{16}$ O. Collet, Étude..., op. cit., p. 124, n. 8 .

${ }^{17}$ Le terme de «fine amor» est d'ailleurs prononcé au v. 7208, en conclusion de l'épisode : «car il par fine amor Cleopatra amoit».

${ }^{18}$ Jean de Thuin, Le Roman de Jules César, éd. cit., v. 6263-6267 et v. 6294-6297.

${ }^{19}$ Ibid., v. 7022-7025.
} 
de la profondeur et de la sincérité de son amour, comme prend soin de le souligner le narrateur:

Si fait molt bien semblant d'ome qui durement et sanz tricherie aint et molt destroitement ${ }^{20}$.

César apparaît ainsi comme un amant vraiment exemplaire, capable de servir de modèle à tout lecteur en lui permettant «de bonté prandre example et bon enseignement », pour reprendre les termes du prologue ${ }^{21}$.

Et cette exemplarité est partagée par Cléopâtre. Non seulement sa beauté est longuement détaillée dans une somptueuse ecphrasis tout à fait conforme à la poétique des romans d'antiquité et largement développée, puisqu'elle s'étend sur trois laisses, mais encore sa sagesse et sa courtoisie sont soulignées de manière récurrente. Après une apparition éblouissante - «ja mes plus bele dame de vos ielz ne verrez », commente le narrateur -, elle est accueillie poliment par César qui, bien qu'il ait changé de couleur à sa vue, "c'est contre li levez et si l'a saluee/ et sele li respont come preuz et senee ${ }^{22} »$. S'ensuit la requête de Cléopâtre, qui demande à César de la protéger contre les vassaux de son frère, puis un dialogue à la fois raffiné et enjoué où chacun fait assaut d'esprit, César cherchant à dire son amour à Cléopâtre et cette dernière feignant de ne voir là qu'un gab, une aimable plaisanterie. La comparaison de la reine d'Égypte avec Hélène, présente chez Lucain et conservée par Jean de Thuin, prend alors un tout autre sens. Dans la Pharsale, qui évoquait «la beauté malfaisante de la Spartiate»(facie Spartana nocenti ${ }^{23}$ ), elle sonnait comme une malédiction; dans le roman elle devient une hyperbole méliorative, même si le rappel antérieur du jugement de Pâris laisse peser une discrète menace et la crainte latente d'un dénouement tragique, d'autant que l'auteur médiéval associe à Hélène une autre femme fatale, «Yseut la roïne» :

Car nature l'ot si par devise formee

c'onques ou mont ne fu dame plus bele nee

se ce ne fu Eleine que Paris ot amee

ou Yseut la roïne, qui Tristan desirree

ot maint jor et por lui mainte paine enduree ${ }^{24}$.

Femme peut-être fatale mais sûrement exemplaire, Cléopâtre semble donc former avec son amoureux César un couple idéal offert à l'admiration du lecteur. Ajoutons qu'ils sont tous deux mis en scène au sein d'une cour très policée qui offre elle aussi le tableau idéal d'une société courtoise,

car avec els asises sont ordeneement damoiseles et dames gentes et de jovent

\footnotetext{
${ }^{20}$ Ibid., v. 7026-7027.

${ }^{21}$ Ibid., v. 17.

${ }^{22}$ Ibid., v. 6113 et v. 6133-6134.

${ }^{23}$ Lucain, La guerre civile (La Pharsale), éd. cit., livre X, v. 61.

${ }^{24}$ Ibid., v. 6038-6042.
} 
sages et enparlees, de bon afaitement, qui servoient la dame, plus en i a de cent $^{25}$.

Comme l'écrit Olivier Collet, «le déploiement de ce réseau intertextuel autour de l'aventure amoureuse de César et de Cléopâtre peut [toutefois] sembler de nature conventionnelle et typologique ${ }^{26}$ «. L'originalité majeure de Jean de Thuin est donc d'insérer au cœur d'un épisode en fait assez convenu un long développement théorique sur l'amour qui couvre près de vingt laisses ${ }^{27}$, n'a rien à voir avec Lucain ni avec les Faits des Romains, et peut dans une certaine mesure donner l'impression d'offrir, comme le note Olivier Collet, de «fortes analogies avec [...] le Roman d'Eneas », bien que sa présence s'explique aussi sans doute par la vogue des Arts d'aimer au $\mathrm{XIII}^{\mathrm{e}}$ siècle $^{28}$. Certes là aussi la topique règne en maître, ce qui enlève d'ailleurs tout espoir d'identifier la source précise de ce développement - si tant est qu'elle existe $^{29}$. Mais l'analyse du sentiment amoureux y présente un certain nombre de caractéristiques voisines de ce qu'on trouve dans les romans d'antiquité du milieu du XII ${ }^{\mathrm{e}}$ siècle, et notamment dans l'Eneas. L'exposé théorique, qui intervient entre le moment où César, frappé par le dard de l'amour, endure dans son lit les tourments de l'insomnie et celui où, après une nuit agitée, il se confie au «chevaliers cortois » qui sera son mandataire auprès de Cléopâtre, s'ouvre en effet sur une tentative de définition de l'amour qui témoigne du désir propre aux «mises en roman» d'appréhender ce phénomène de manière objective, avec lucidité, dans une approche presque clinique. C'est ainsi que la «fine amor» est définie comme un processus à la fois physiologique et psychologique où la volonté du cœur est fortement tributaire des désirs du corps, qui recherche avant tout son plaisir :

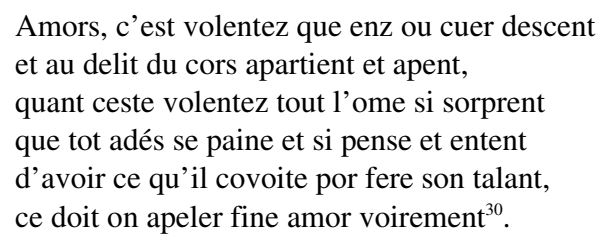

La suite immédiate du développement, consacrée à une analyse minutieuse du rôle respectif de l'œil, qui perçoit la beauté, et du cœur, qui en recevant cette image donne naissance à la pensée, confirme le projet d'une approche presque scientifique du phénomène amoureux, d'autant que ces premières laisses sont construites sur une structure dialogique qui fait résonner deux voix, celle du narrateur qui enseigne et une autre voix mal identifiée, peut-être celle d'un lecteur ou d'un auditeur potentiel, qui interroge et se met ainsi dans la posture d'un élève curieux mais obéissant :

\footnotetext{
${ }^{25}$ Jean de Thuin, Le Roman de Jules César, éd. cit., v. 7006-7009.

${ }^{26}$ O. Collet, Étude..., op. cit., p. 125.

${ }^{27}$ Dix-neuf exactement, du v. 6362 au v. 6766 du Roman.

${ }^{28}$ O. Collet, Étude..., op. cit., p. 130.

${ }^{29}$ Voir à ce sujet la longue note d'O. Collet, Étude..., op. cit., p. 130-131, note 12.

${ }^{30}$ Jean de Thuin, Le Roman de Jules César, éd. cit., v. 6362-6367.
} 


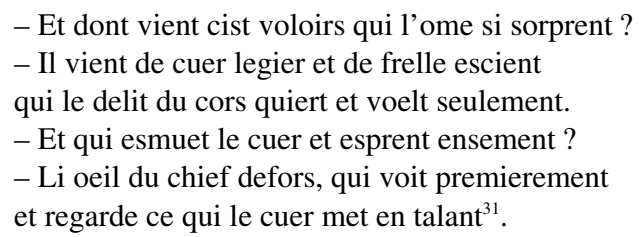

Cette structure dialogique peut en effet évoquer les dialogues à portée didactique qui mettent aux prises l'épouse du roi Latinus et sa fille Lavine, voire les dialogues intérieurs d'Eneas et de Lavine. Cela d'autant mieux que si le désir du corps est présenté comme premier, Jean de Thuin prend bien garde de réduire l'amour à cela. La suite de son développement, en démontrant d'abord que la beauté n'est pas la seule à susciter l'amour, qui peut naître aussi de la «cortoisie » et de la «valors $»^{32}$, puis que cet amour, si enclin qu'il soit spontanément à la démesure, peut et doit se vivre avec mesure et raison ${ }^{33}$, introduit peu à peu l'idée d'une discrète ascèse du sentiment amoureux, fondée sur toute une série d'exemples et de contre-exemples traditionnels : il faut savoir, dit le narrateur, dissimuler sa passion, faire preuve de retenue et de loyauté, fuir la fourberie et la jalousie, la leçon étant d'ailleurs valable tant pour l'ami que pour l'amie. On peut ainsi être tenté de conclure, comme Olivier Collet, que «la fable et l'idéologie amoureuse [du] poème [de Jean de Thuin] rencontrent la spiritualité figurée par les romans antiques et arthuriens ${ }^{34} »$.

Quelques fausses notes laissent toutefois planer un certain malaise, et incitent à s'interroger. D'abord l'exposé théorique du narrateur est, il faut bien le dire, assez mal intégré au contexte narratif. Dans l'Eneas les considérations didactiques étaient habilement intégrées au discours des protagonistes. Rien de tel dans notre roman, où cet exposé est clairement présenté comme une digression du narrateur, la présence récurrente d'un je auctorial semblant même vouloir affirmer, derrière ce narrateur, la présence et l'autorité de Jean de Thuin en personne. Le développement sur l'amour s'ouvre en effet sur ces mots :

Et por tant que d'amors ont regnié et parlé plusor, si m'estuet dire ce que j'en ai pensé ${ }^{35}$.

Présent dès l'ouverture du discours théorique, le je préside aussi à sa clôture à travers deux vers conclusifs, avant de revenir à la narration proprement dite :

\footnotetext{
${ }^{31}$ Ibid., v. 6368-6373.

${ }^{32}$ Ibid., v. 6410-6411 : «biautez, c'est ochoisons qui fet amer plusors/ les autres fet amer cortoisie et valors ».

${ }^{33}$ Ibid., v. 6433-6441 : «bien di que savoir gist et mesure en amer/ nonporquant j'ai oï les plusors ramembrer qu'en ne puet en amors sens ne reson garder [...]/ et qui i velt par sens et par mesure errer/ si dit on qu'il n'est pas fins amanz sans fauser ;/ mes je di c'on doit bien par mesure mener/ et par sens son amor et par reson ouvrer».

${ }^{34}$ O. Collet, Étude..., op. cit., p. 125.

${ }^{35}$ Jean de Thuin, Le Roman de Jules César, éd. cit., v. 6357-6358.
} 
Or vos ai dit partie de ce qu'il me sembloit

d'amors et an quel point homs sages amer doit ${ }^{36}$.

De ce fait, comme le reconnaît du reste Olivier Collet, ce discours «alerte par l'absence d'une conciliation véritable entre son sujet et son objet. La passion déclenchée subitement en César s'en donne comme le prétexte, mais le comportement amoureux de ce dernier n'en constitue pas la référence, sinon lointaine. Il n'y a après tout entre la théorie de l'auteur et le déroulement de l'épisode que des interactions tout à fait superficielles ${ }^{37}$ ». Cela d'autant mieux que deuxième fausse note - l'idéalisation de César et de Cléopâtre contraste assez nettement avec plusieurs considérations fortement désenchantées dont se nourrit l'exposé théorique du narrateur. Ainsi, après avoir soigneusement défini le comportement exemplaire qu'il convient d'avoir en amour («et ainsi com j'ai dit covient il sans fauser/ tenir sens et reson et mesure en amer»), ce dernier poursuit, sur un ton désabusé :

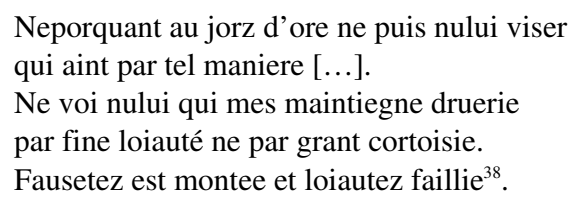

Et un peu plus loin il conclut, en passant du désenchantement à la plainte accusatrice :

Molt me plaing q'amor bone est si du tot perie
et qu'ele a si perdue la grande seignorie
qu'elle souloit avoir as gens d'ancesserie ${ }^{39}$.

Bien sûr nous avons là encore la mise en œuvre d'un topos, comparable à celui qu'on peut trouver par exemple dans le prologue du Chevalier au Lion quand Chrétien de Troyes, opposant le passé au présent, met en regard l'exemple de parfaite courtoisie qu'offre selon lui la cour du roi Arthur et la dégénérescence des mœurs de son temps, où Amours, «mout abaissie», «est tournee a fable ${ }^{40} »$. De même l'opposition établie par Jean de Thuin entre ses contemporains et les "gens d'ancesserie», les gens du passé, illustrés par l'exemple de Piramus et Tisbé, pourrait avoir pour fonction de souligner a contrario l'exemplarité du couple formé par César et Cléopâtre. Mais sous l'apparent topos peuvent aussi se glisser des intentions plus subtiles, comme nous le prouve Chrétien en mettant en scène, juste après son prologue, des personnages dont le comportement déplacé et l'arrogante

${ }^{36}$ Ibid., v. 6762-6763.

${ }^{37}$ O. Collet, Étude..., op. cit., p. 131.

${ }^{38}$ Jean de Thuin, Le Roman de Jules César, éd. cit., v. 6544-6545 et v. 6550-6555.

${ }^{39}$ Ibid., v. 6684-6686.

${ }^{40}$ Chrétien de Troyes, Le Chevalier au Lion, éd. et trad. D. Hult, Paris, Le Livre de Poche, coll. «Lettres Gothiques », 1994, v. 20 et 24. 
grossièreté démontrent que la cour d'Arthur, en fait, n'est pas précisément un modèle de courtoisie ${ }^{41}$. On peut donc se demander si une stratégie analogue n'est pas à l'œuvre ici et reconsidérer, sur la base de ces quelques fausses notes, l'apparente exemplarité des amours de César: est-il vraiment un nouvel Énée, magnifié par l'amour, et Cléopâtre ne serait-elle pas en fait une fausse Lavine ?

Outre l'idéalisation dont bénéficie la reine d'Égypte dans le roman de Jean de Thuin, plusieurs détails semblent bel et bien, à première vue, vouloir l'apparenter à la célèbre amoureuse du Roman d'Eneas. En parfaite contradiction avec le texte de Lucain, l'accent est mis notamment à plusieurs reprises sur sa jeunesse, son innocence et sa pudeur. Ainsi lorsque César, lors de leur premier entretien, cherche à lui déclarer son amour, elle feint de ne voir là qu'une plaisanterie et réplique : «se je sui jone et simple, por tant ne me gabe $^{42} »$. Et un peu plus loin, lorsque le chevalier envoyé par César la surprend dans son lit, «tretote eschevelee », le narrateur note la rougeur qui trahit son pudique embarras : «la dame li respont, s'a la color muee/ por ce qui l'a ainsi en son esveil trovee ${ }^{43} »$. Mais cette apparente ingénue sait aussi se comporter, en faisant preuve d'une très grande maîtrise de soi, d'une manière parfaitement conforme à ses intérêts. Ainsi, après avoir répondu très courtoisement à la requête qu'elle lui a présentée, César, qui commence à être séduit, la prend par la main, la fait asseoir auprès de lui et lui donne un baiser.

Et cele est tant senee que point ne s'a ganchie,

ainz fet semblant defors qu'en son cuer en soit lie ${ }^{44}$.

On sait que si le «semblant» renvoie à l'apparence, cette dernière peut être tout à fait conforme à l'intériorité et à la vérité des sentiments : elle n'est pas forcément mensongère. La présence de l'adverbe «defors", qui insiste sur le fait que cette apparence est avant tout extérieure, laisse toutefois planer un doute sur la sincérité de l'héroïne. N'oublions pas que Jean de Thuin, contemporain de Jean de Meun, connaît peut-être Faux-Semblant. En ne se dérobant pas au baiser donné par César, Cléopâtre semble mettre en place les premiers éléments d'une stratégie destinée à lui assurer la bienveillance active de celui dont elle veut se faire un allié contre son frère, d'autant que tout le reste de son comportement témoigne de la même habileté manœuvrière. Ainsi, après avoir feint de ne voir dans les premières déclarations de César qu'une plaisanterie, et s'être retirée comme pour mieux attiser son désir ${ }^{45}$, elle accueille très favorablement son envoyée ${ }^{46}$, accepte sans grande hésitation, après quelques réticences assez convenues, une deuxième entrevue, et lors de ce second entretien, sait ouvrir avec aisance le dialogue qui conduira très vite à l'aveu

\footnotetext{
${ }^{41}$ Voir entre autres le sénéchal Keu, chef-d'œuvre de discourtoisie.

${ }^{42}$ Jean de Thuin, Le Roman de Jules César, éd. cit., v. 6235.

${ }^{43}$ Ibid., v. 6838-6839.

${ }^{44}$ Ibid., v. 6199-6200.

${ }^{45}$ Ibid., v. 6261-6262 : « et aprés ces paroles la dame o le vis cler/ arriere s'en retorne, n'i volt plus demorer».

${ }^{46}$ Ibid., v. 6896-6897 : «Sire, voir! dit la dame, ja nel quier refuser/ amor de haut baron qui tant fet a loer!».
} 
réciproque : «lors l'en a aresnié bel et cortoisement ${ }^{47}$ ». Nous sommes ici très loin de Lavine, l'amoureuse timide qui avoue certes son amour dans une lettre mais n'adresse jamais la parole à son ami, ne dialoguant qu'avec elle-même dans de longs monologues solitaires où elle se débat contre un amour interdit.

D'autant plus que dans la scène de l'aveu, l'habileté de Cléopâtre, qui sait accepter tout en se dérobant, offre un superbe exemple d'hypocrisie tactique qui dissimule mal un calcul très cynique. À César qui lui avoue assez crûment son désir en lui demandant la «joie d'amors »

et le delit qui est a amant plus plaisanz

est ce quant ens ez braz de s'amie est gisanz ${ }^{48}$,

elle répond sans trop s'émouvoir en le renvoyant à la décision de son chambellan :

Sire, ce dit la dame, vos estes requeranz

chose qui molt est grande et qui molt sui dotanz,

car je dout qu'a tel chose n'ert pas bien asentans

mes chamberlans qui m'est par bone foi gardanz

et qui mes mestres est et a droit conseillant ;

mes se cil le me loë, ja n'iere refusanz

que volentiers ne doie fere toz vos talanz ${ }^{49}$ !

César, qui comprend sans peine ce que parler veut dire, acquiesce et va tout aussitôt trouver le chambellan, à qui il fait des promesses précises qui emportent bien vite son adhésion :
et si li dit q'avoir a plenté li donra
et de tote sa guerre si bien li aidera
que sa dame l'onor d'Egypte retanra
et li bailli de toute la contree fera.
Tant a Cesar pramis et tant li enorta
que li chamberlans tot son bon li otroia
por l'onor et l'avoir que il molt covoita ${ }^{50}$.

La scène d'amour débouche ici sur la «covoitise» de «l'avoir», c'est-à-dire sur un marchandage pur et simple que la reine d'Égypte ne mène pas personnellement, mais qu'elle délègue à un subordonné qu'on peut bien soupçonner d'être son complice. Et pourtant Cléopâtre, dans son entretien préliminaire avec le chevalier envoyé par César, avait affirmé ne pas vouloir vendre son amour, et être plus sensible à la valeur personnelle de son soupirant qu'à toutes les promesses et à tous les dons qu'il pourrait vouloir lui faire :

\footnotetext{
${ }^{47}$ Ibid. , v. 7031.

${ }^{48}$ Ibid., v. 7072-7073.

${ }^{49}$ Ibid., v. 7074-7080.

${ }^{50}$ Ibid., v. 7091-7097.
} 


\begin{abstract}
Mes de tant me veil bien envers vos escuser que plus me plait en lui ce qu'il se fait nomer de proesce et de sens, en li n'a que blasmer, que ce qu'il me porroit prometre ne doner, car n'est pas marcheande m'amor por acheter ${ }^{51}$ !
\end{abstract}

Rapportée à ce qui suit, cette fière affirmation n'apparaît plus que comme le discours mensonger d'une femme cynique et rusée qui sait dissimuler ses vues intéressées derrière des stratégies habiles et diversifiées. Voilà qui permet d'établir un écho avec le discours théorique du narrateur lorsque, après avoir condamné le manque de loyauté des hommes, il ajoute qu'on peut bien les «escuser en partie», «car en voit as jorz d'ore mainte fame a la fie/ qui s'entremet ausi d'amer par fauserie $^{52} »$, avant de faire défiler sur trois longues laisses le cortège des femmes perfides, des femmes intéressées ( «ce sont celes qui sont lor amors otroianz/ par promesse et par dons quant cil lor est proianz ») et des infidèles coquettes ${ }^{53}$. Un détail formel peut d'ailleurs ici retenir notre attention. Ces trois laisses dénonciatrices sont curieusement construites sur la même assonance, en -ant (pour la première) ou en anz (pour les deux autres). Or la scène d'aveu qui débouche sur un marchandage cynique est elle aussi construite sur une assonance en -anz, comme si Jean de Thuin avait voulu, par cet écho phonique, attirer notre attention sur la parenté latente qui unit les deux passages.

En dépit des apparences, Cléopâtre peut donc difficilement prétendre être une autre Lavine. Et César n'est pas davantage, à notre avis, un nouvel Eneas. Ce dernier était, ne l'oublions pas, présenté dans le roman qui porte son nom comme un personnage assez louche qui arrivait en Italie, s'il faut en croire la mère de Lavine, précédé par une réputation détestable, celle d'un «traïtor» doublé d'un « sodomite ${ }^{54}$. Mais ce mauvais garçon était régénéré, purifié par l'amour ${ }^{55}$. César, présenté par Jean de Thuin, dans l'ensemble, sous un jour mélioratif comme un guerrier émérite, n'a pour sa part nul besoin d'être régénéré. Il ne semble donc demander à l'amour, en fait, qu'une courtoisie de façade qui dissimule assez mal la crudité d'un désir essentiellement sensuel, comme on peut le voir d'ailleurs à travers la franche brutalité de l'aveu que nous avons cité plus haut ${ }^{56}$. C'est sans doute pourquoi le portrait de Cléopâtre, si topique et mélioratif qu'il soit, présente bien des traits qui insistent avant tout sur la sensualité qui en émane (les cheveux dénoués -

\footnotetext{
${ }^{51}$ Ibid., v. 6898-6902.

${ }^{52}$ Ibid., v. 6568-6570.

${ }^{53}$ Ce sont les laisses 364, 365 et 366 du Roman; les vers cités sont les v. 6602-6603. Jean de Thuin ajoute de manière significative que ces femmes intéressées sont celles «qui plus sont decevanz/ et qui plus s'entremetent de mostrer fauz semblanz » (v. 6600-6601).

${ }^{54}$ Eneas, éd. cit., t. II, v. 8583.

${ }^{55}$ Comme l'a montré R. J. Cormier, One heart one mind: the rebirth of Virgil's hero in medieval french romance, University of Mississipi, 1973, surtout dans ses deux derniers chapitres (p. 229-286 : the tower of love/ ordo novus).

${ }^{56}$ On peut alors soupçonner le «semblant» d'amant courtois qu'il montre de n'être qu'un faux-semblant (cf. les v. 7026-7027, cités plus haut).
} 
un souvenir de $\operatorname{Lucain}^{57}$-, la gorge blanche, la bouche faite pour le baiser ${ }^{58}$ ), et se clôt sur l'affirmation désinvolte que le plus grand plaisir serait de la tenir nue entre ses bras,

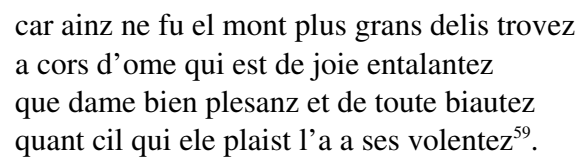

C'est aussi pourquoi la description de l'union physique entre les deux amants se conclut par une métaphore érotico-ludique qui évoque d'une manière assez précise la défloration de l'héroïne :

Si ne sai que je plus de lor estre vos die, car je cuit que ainçois que la nuit soit faillie que la dame avera qui qu'il en prende envie tresperciee la porte que a l'entrer ne crie $^{60}$.

Et là aussi on voit s'établir un écho entre la narration et l'exposé théorique qui place très nettement à l'origine de l'amour, nous l'avons vu, le désir de la jouissance charnelle ${ }^{61}$

En dépit des apparences, Jean de Thuin n'idéalise donc pas vraiment ses deux héros : l'amour est chez eux moins spirituel et plus physique, voire plus cynique, que chez leurs prédécesseurs du Roman d'Eneas. On aurait tort toutefois, croyons-nous, de penser qu'il les blâme ou qu'il les désapprouve. Les épithètes valorisantes dont il les couvre recèlent peut-être un brin d'ironie, mais pas plus. Même si l'amour qu'il porte à Cléopâtre est essentiellement sensuel, César reste un «ber» (v. 7035), un vaillant guerrier, et il ne trompe pas son amie, puisqu'il sait tenir ses promesses. Il avait promis au chambellan «que sa dame l'onor d'Egypte retanra» (v. 7093), et il tient parole, puisqu'avant de rentrer à Rome

si a de toute Egipte la couronne donee

Cleopatra la bele que il avoit amee :

ensement fu la dame roïne couronnee,

si li a bien Cesar s'amor guerredonnee ${ }^{62}$.

\footnotetext{
${ }^{57}$ Jean de Thuin, Le Roman de Jules César, éd. cit., v. 6065-6066 : « de ces garnemenz est la dame bien paree/ mes de guimple n'ot point, ainz ert eschevelee». Lucain l'avait dépeinte «les cheveux en désordre comme si elle les avait arrachés » (ueluti laceros dispersa capillos), pour mieux apitoyer César (La Pharsale, éd. cit., livre X, v. 84).

${ }^{58}$ Ibid., v. 6093-6095 et v. 6102-6103.

${ }^{59}$ Ibid., v. 6122-6125.

${ }^{60}$ Ibid., v. 7179-7182. Sur cette métaphore, voir la note d'O. Collet, éd. cit., p. 474.

${ }^{61}$ Cf O. Collet (Étude..., op. cit., p. 131-132), qui loue Jean de Thuin d'avoir mis en évidence «la souveraine puissance du désir et de la libido».

${ }^{62}$ Jean de Thuin, Le Roman de Jules César, éd. cit., v. 7761-7764.
} 
C'est sans doute pour cette raison que son amour est qualifié malgré tout de «fine amor» (v. 7208). Quant à Cléopâtre, ce n'est pas sans raison que le narrateur la dit «preuz et senee»(v.6134). Même si l'amour qu'elle porte à César est en bonne partie intéressé, elle sait en effet mener ses affaires avec une grande maîtrise du discours et une habileté consommée que l'exposé théorique, en fait, est loin de désapprouver. Après s'être plaint qu'amour ait «si perdue la grande seignorie/ qu'elle souloit avoir as gens d'ancesserie » et avoir cité comme modèle exemplaire d'amour du passé l'histoire de «Piramus qui s'ocist por s'amie/ Tysbé, qui autresi perdi por lui sa vie», le narrateur poursuit en effet :

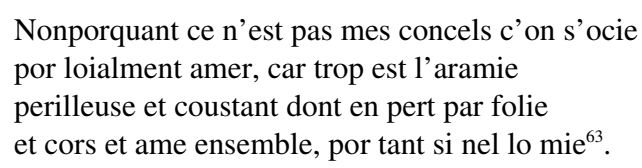

Esprit modéré, Jean de Thuin ne préconise donc pas les fins tragiques. Cela ne peut que le rendre indulgent envers une Cléopâtre qui, contrairement à Hélène de Troie et contrairement à ce qu'affirme Lucain, ne porte chez lui malheur ni à l'Égypte ni à Rome, et qui sait aussi éviter le suicide dont est victime Didon délaissée par Énée. En la rétablissant dans ses droits sur le trône d'Égypte, César réalise en fait une heureuse opération dont bénéficie toute la contrée, puisqu'il met fin aux guerres fratricides qui opposaient Cléopâtre à son frère et à sa sœur Arsinoé :

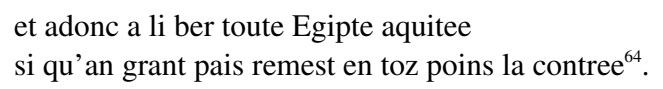

Mais évidemment le couple de ses héros s'éloigne ici du modèle mis en scène par l'Eneas, celui d'un couple uni par un amour à la fois charnel et spirituel qui rayonne sur tout le pays dont ils deviennent conjointement les souverains. Contrairement à Eneas couronné avec Lavine, César rentre seul et triomphe seul à Rome, ce qui est d'ailleurs conforme à la vérité historique :

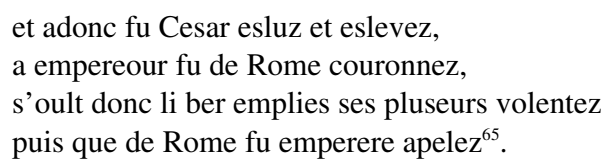

En dépit de leur habillage courtois, les amours de César et de Cléopâtre évoquent donc moins l'union d'Eneas et de Lavine que la brève aventure d'Alexandre avec la reine Candace, et c'est peut-être pour cette raison que Jean de Thuin a pris comme modèle formel le Roman d'Alexandre. La tradition postérieure, illustrée par le Livre

\footnotetext{
${ }^{63}$ Ibid., v. 6685-6686, v. 6689-6690 et v. 6691-6694.

${ }^{64}$ Ibid., v. 7765-7766.

${ }^{65}$ Ibid., v. 9390-9393 (fin de l'avant-dernière laisse, juste avant l'épilogue). César n'a pas été, on le sait, couronné empereur, mais il a bel et bien triomphé de ses rivaux et exercé le pouvoir suprême à Rome avant son assassinat - dont Jean de Thuin ne parle pas.
} 
du Cơur de René d'Anjou, ne s'y est d'ailleurs pas trompée. Alors qu'Eneas, dans ce livre, est représenté aux côtés de Créüse puis de Lavine, ses deux épouses légitimes ${ }^{66}$, César y est figuré, nous l'avons vu, sous la dépendance d'une Cléopâtre pour qui il aurait «habandonné tout pris et los d'onneur/ conquestes et vaillances et les nobles faiz d'armes ${ }^{67}$ », une Cléopâtre qui l'aurait pour l'éternité retenu auprès d'elle en Égypte : fantasme énéen à coup sûr, qui fait de la reine orientale une Didon triomphante, mais fantasme qui donne à voir César, sans égard pour la vérité historique, comme un Énée bridé et amenuisé, bref moins un nouvel Énée qu'un faux Énée, dans une perspective sans doute assez ludique.

Il est temps de conclure. Le souvenir d'Eneas et de ses deux amantes, Didon puis Lavine, plane bien sur le Roman de Jules César, ne serait-ce qu'à travers l'idéalisation courtoise dont bénéficient les héros et les réflexions méthodiques du narrateur sur la nature de l'amour. Mais du milieu du XII ${ }^{\mathrm{e}}$ au milieu du XIII ${ }^{\mathrm{e}}$ siècle, le contexte culturel a changé. On a vu d'abord apparaître un souci plus exigeant de la vérité historique dont le prologue du roman de Jean de Thuin rend témoignage : il répète à plusieurs reprises qu'il veut relater la «verté de l'estoire» ou «la vraie estoire » de Rome ${ }^{68}$. Même si ces affirmations ont quelque chose de topique, elles ne sont pas seulement conventionnelles et gratuites. Jean de Thuin aurait pu, en s'inspirant par exemple de Suétone, bien connu au Moyen Age et exploité par les Faits des Romains, faire venir Cléopâtre à Rome, voire la faire couronner aux côtés de César. Il s'y est refusé, par égard pour «l'estoire vraie ${ }^{69} »$. D'autre part, chez ce contemporain de Rutebeuf et de Jean de Meun, la «fine amor » n'a plus sans doute le bel enthousiasme des commencements. Jean de Thuin tient à en réaffirmer les principes et l'attachement qu'il leur porte, mais il tient aussi à souligner les difficultés que rencontre leur mise en ouvre dans la réalité pratique des rapports humains. Avec beaucoup de finesse, toutefois, il s'abstient de trancher. Nous avons vu que deux lectures étaient possibles, une lecture démystifiante, une lecture plus méliorative, et que ces deux lectures pouvaient trouver un appui dans un exposé théorique en fait très nuancé, et suffisamment détaché du contexte narratif pour ne pas paraître le commander. Les marques dont Jean de Thuin a parsemé son texte sont assez subtiles pour laisser au lecteur un large espace de libertét ${ }^{70}$. Aurait-il par hasard prévu deux publics, un public seigneurial et laïque avant tout friand de

\footnotetext{
${ }^{66}$ René d'Anjou, Le Livre du Cæeur d'amour épris, éd. cit., § XC, v. 1285-1290 : «car Creüsa me fut la premiere contrainte/ pour laquelle plouray depuis des larmes mainte [...]/ puis l'amour de Lavigne, fille au roi Latin, fu/ cause dont j'ay ycy le mien blazon pendu ».

${ }^{67} \mathrm{Ibid}$., § LXXXIII, v. 1216-1217 (cf. le passage cité plus haut).

${ }^{68}$ Jean de Thuin, Le Roman de Jules César, éd. cit., v. 35 et v. 62.

${ }^{69}$ Ibid., v. 3. Sur la naissance d'une conscience historique au XIII ${ }^{\mathrm{e}}$ siècle, voir C. CroizyNaquet, Écrire l'histoire romaine au début du XIII siècle, Paris, Champion, 1999. Dans le $\S$ LII du livre I de sa Vie des douze Césars, Suétone écrit que la plus grande passion de César fut pour Cléopâtre et que, l'ayant fait venir à Rome, il ne la renvoya que comblée d'honneurs et de récompenses magnifiques.

${ }^{70}$ Cf. O. Collet, Étude..., op. cit., p. 132 : «le discours théorique ne présente donc pas une grille de lecture par laquelle il conviendrait obligatoirement d'interpréter le comportement des personnages, il n'exige pas qu'on les juge d'après le 'canon' idéologique de la courtoisie ».
} 
normes courtoises, et un public de clercs beaucoup moins favorables à la figure de Jules César? En l'absence de toute information sur les commanditaires éventuels de l'ouvrage, l'hypothèse reste malheureusement invérifiable. Ce qui est sûr, toutefois, c'est que le modèle énéen dont il s'inspire lui permet de valoriser ses héros en corrigeant l'approche trop dépréciative de Lucain, ce qui est conforme à son dessein d'ensemble; mais il maintient en même temps, non sans habileté, l'écart avec lui, pour répondre aux exigences d'une nouvelle écriture, l'écriture historique, dont dès son prologue il s'affirme tributaire.

Francine MORA

Université de Versailles - Saint-Quentin-en-Yvelines 